\title{
Perinatal bisphenol A exposure promotes dose-dependent alterations of the mouse methylome
}

\author{
Jung H Kim ${ }^{1,2}$, Maureen A Sartor ${ }^{2}$, Laura S Rozek ${ }^{1,3}$, Christopher Faulk', Olivia S Anderson ${ }^{1}$, Tamara R Jones ${ }^{1}$,
} Muna S Nahar ${ }^{1}$ and Dana C Dolinoy ${ }^{1 *}$

\begin{abstract}
Background: Environmental factors during perinatal development may influence developmental plasticity and disease susceptibility via alterations to the epigenome. Developmental exposure to the endocrine active compound, bisphenol A (BPA), has previously been associated with altered methylation at candidate gene loci. Here, we undertake the first genome-wide characterization of DNA methylation profiles in the liver of murine offspring exposed perinatally to multiple doses of BPA through the maternal diet.

Results: Using a tiered focusing approach, our strategy proceeds from unbiased broad DNA methylation analysis using methylation-based next generation sequencing technology to in-depth quantitative site-specific CpG methylation determination using the Sequenom EpiTYPER MassARRAY platform to profile liver DNA methylation patterns in offspring maternally exposed to BPA during gestation and lactation to doses ranging from 0 BPA/kg (Ctr), $50 \mu \mathrm{g}$ BPA $/ \mathrm{kg}(\mathrm{UG})$, or $50 \mathrm{mg}$ BPA $/ \mathrm{kg}(\mathrm{MG})$ diet ( $\mathrm{N}=4$ per group). Genome-wide analyses indicate non-monotonic effects of DNA methylation patterns following perinatal exposure to BPA, corroborating previous studies using multiple doses of BPA with non-monotonic outcomes. We observed enrichment of regions of altered methylation (RAMs) within CpG island (CGI) shores, but little evidence of RAM enrichment in CGIs. An analysis of promoter regions identified several hundred novel BPA-associated methylation events, and methylation alterations in the Myh7b and S/c22a12 gene promoters were validated. Using the Comparative Toxicogenomics Database, a number of candidate genes that have previously been associated with BPA-related gene expression changes were identified, and gene set enrichment testing identified epigenetically dysregulated pathways involved in metabolism and stimulus response.
\end{abstract}

Conclusions: In this study, non-monotonic dose dependent alterations in DNA methylation among BPA-exposed mouse liver samples and their relevant pathways were identified and validated. The comprehensive methylome map presented here provides candidate loci underlying the role of early BPA exposure and later in life health and disease status.

Keywords: Bisphenol A, DNA methylation, Environmental epigenomics, MethylPlex

\section{Background}

The early developmental environment is an influential predictor of subsequent phenotypes and disease risk later in life. A growing body of work supports the "developmental origins of health and disease" (DOHaD) hypothesis, which posits that chemical and/or nutritional influences during early life result in long-lasting effects

\footnotetext{
*Correspondence: ddolinoy@umich.edu

'Department of Environmental Health Sciences, University of Michigan, 1415

Washington Heights, Ann Arbor, Michigan, USA

Full list of author information is available at the end of the article
}

and point to epigenetic inheritance as a prime mechanism $[1,2]$. Epigenetic modifications, such as DNA methylation and chromatin markings, are established early in development and can shape susceptibility to disease, resulting in diverse phenotypes among genetically identical individuals [3]. Until recently, however, most attempts to elucidate the effects on the epigenome following environmental and nutritional exposures were either candidate gene driven or based on epigenetic techniques with limited genome coverage/sensitivity. Using bisphenol A (BPA) as a representative early environmental exposure alongside an

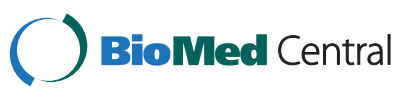

(c) 2014 Kim et al.; licensee BioMed Central Ltd. This is an open access article distributed under the terms of the Creative Commons Attribution License (http://creativecommons.org/licenses/by/2.0), which permits unrestricted use, distribution, and reproduction in any medium, provided the original work is properly cited. 
established dose-dependent mouse model of perinatal exposures [4-7], we have developed a comprehensive strategy for evaluating environmental effects on the developing epigenome (Figure 1).

BPA is a high-production volume monomer used in the manufacture of polycarbonate plastic and epoxy resins. It is present in products that are routinely used, including food and beverage containers, baby bottles, dental composites, and thermal receipt paper [8]. Several studies have reported detectable levels of total urinary BPA in a large proportion of populations around the world [9-11]. An evaluation of circulating blood BPA levels in pregnant women in southeast Michigan indicated exposure levels between $0.5 \mu \mathrm{g} / \mathrm{L}$ and $22.3 \mu \mathrm{g} /$ $\mathrm{L}($ mean $5.9 \mu \mathrm{g} / \mathrm{L}$ ) [12], and our recent study of human fetal liver samples indicated that there is considerable exposure to BPA during pregnancy and that BPA in fetuses was in a unconjugated form not readily eliminated from the body [13]. These findings indicate that in utero development and infancy may be particularly vulnerable time periods for exposure to BPA.

Toxicology studies indicate BPA exposure, both at high levels and levels well below the established U.S. Environmental Protection Agency (EPA) reference dose of $50 \mu \mathrm{g} / \mathrm{kg}$ body weight/day, results in a variety of physiological changes implicated in breast and prostate

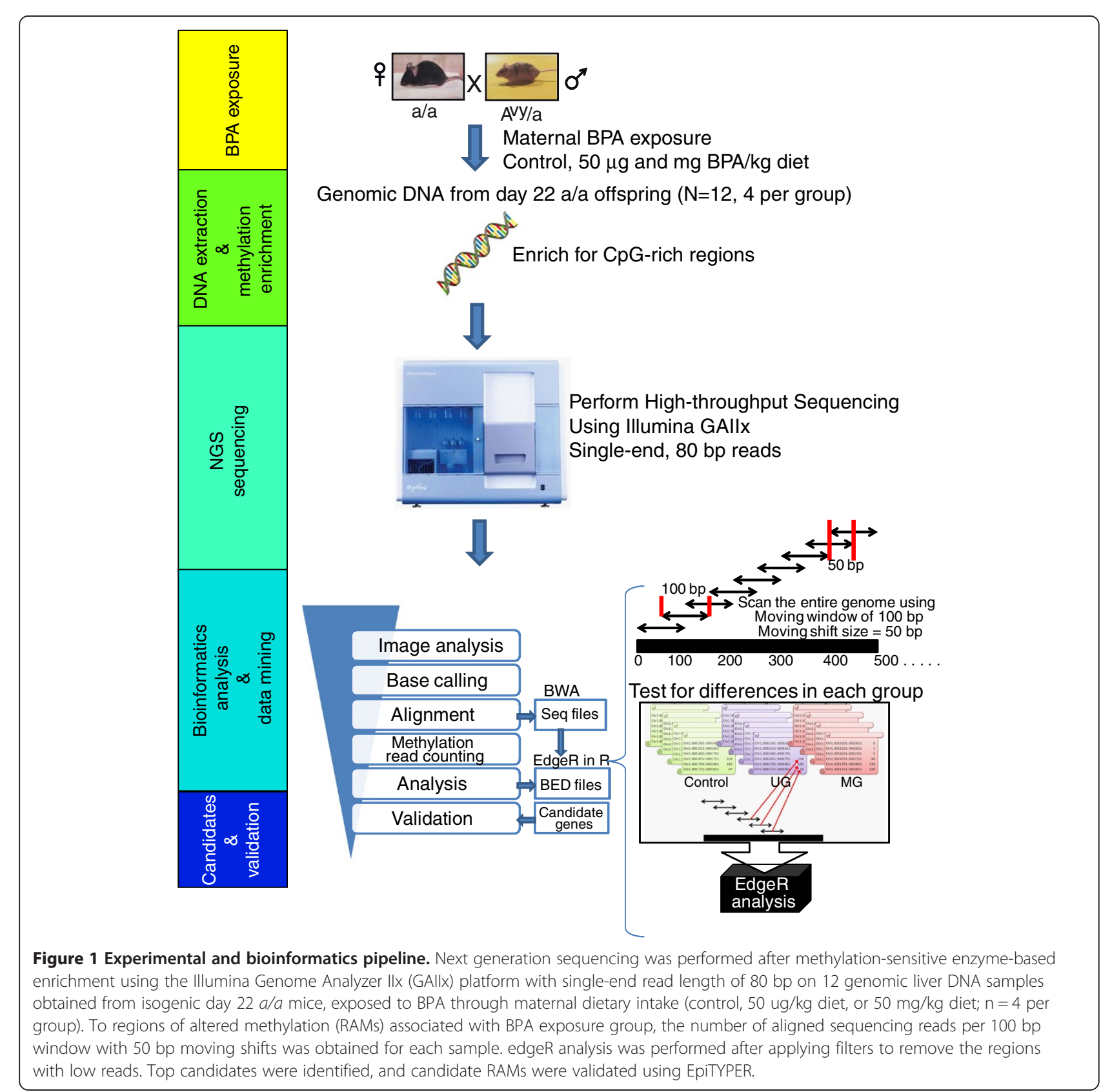


cancer, reproductive dysregulation, and behavioral abnormalities $[14,15]$. Epidemiology studies have described associations between increased BPA levels with cardiovascular disease risk, decreased semen quality, altered childhood behavior, and recurrent miscarriages [16-19]. BPA can mimic or antagonize endogenous hormones by binding weakly to steroid receptors including estrogen receptors (ER $\alpha$ and $\beta$ ) and thyroid hormone receptor [20-22]. BPA also binds strongly to the trans-membrane ER, G proteincoupled receptor 30 (GPR30), as well as the orphan nuclear receptor estrogen related receptor gamma (ERR $\gamma$ ) [23,24], and can also activate transcription factors, including peroxisome $\mathrm{x}$ receptor (PXR) and aryl hydrocarbon receptor $(\mathrm{AhR})$, which can dimerize with steroid receptors $[25,26]$.

BPA is associated with epigenetic alterations following developmental exposures [4,5,27-30]. In a rat model, Ho and colleagues observed multiple changes in genespecific DNA methylation patterns in the adult male prostate, including hypomethylation of the phosphodiesterase type 4 variant 4 (Pde4d4) [27]. Hypomethylation of the nucleosome binding protein-1 (Nsbp1) gene promoters and hypermethylation of the hippocalcin-like 1 (Hpcal1) gene promoter was also reported in rats following neonatal exposure to low concentrations of BPA $(10 \mu \mathrm{g} / \mathrm{kg}$ of body weight BPA) [30]. Altered methylation and subsequent aberrant gene expression was associated with a marked increase in prostate cancer risk. Using the viable yellow agouti $\left(\mathrm{A}^{\mathrm{vy}}\right)$ mouse model, we have shown that maternal dietary exposure to moderate levels of $\mathrm{BPA}$ (50 mg BPA $/ \mathrm{kg}$ diet) resulted in decreased DNA methylation at the $A^{v y}$, and $C a b p^{I A P}$ metastable epialleles $[4,5]$, while exposure to lower doses (50 ng and $50 \mu \mathrm{g} \mathrm{BPA} /$ $\mathrm{kg}$ diet) led to hypermethylating effects at these candidate loci [5]. Finally, using restriction-enzyme based methylation technology, Yaoi and colleagues reported both hyper- and hypomethylation at a methylation-sensitive NotI loci in murine offspring forebrain following gestational exposure to $20 \mu \mathrm{g} / \mathrm{kg}$ body weight of BPA [28]. Recently, the differential methylation in imprinting control regions was reported in maternally BPA-exposed mouse embryos and placentas using pyrosequencing technology. This change in methylation also resulted in abnormal expression in placenta and abnormal placental development [31].

Capitalizing on advances in whole-genome epigenomic and high-throughput quantitative DNA methylation technologies, we developed a comprehensive approach to identify the constellation of genomic loci with altered epigenetic status following dose-dependent perinatal BPA exposure. Using a tiered focusing approach, our strategy proceeded from unbiased broad DNA methylation analysis using methylation-based next generation sequencing technology to in-depth quantitative site-specific CpG methylation determination using the Sequenom EpiTYPER MassARRAY platform. We compared the regions of altered methylation (RAMs) following BPA exposure using bioinformatics and biostatistics methods, and the cellular pathways in which the genes with nearby RAMs function.

\section{Results \\ Analysis pipeline and quality control for identifying differential methylation}

We used the MethylPlex-Next Generation Sequencing (M-NGS) platform to evaluate genome-wide alterations in DNA methylation following perinatal BPA exposure in mice, which requires minimal DNA input ( 50 nanograms) and enriches methylated DNA using a cocktail of methylation-dependent restriction enzymes prior to deep sequencing (Figure 1). Following alignment to the reference mouse genome (version $\mathrm{mm} 9$ ), we confirmed that MethylPlex library reads were enriched in genomic regions containing higher numbers of genes and $\mathrm{CpG}$ islands (CGIs) (Additional file 1: Figure S1). For initial standardization of the data analysis pipeline, we employed a sex-based analysis comparing methylation profiles on chromosomes $\mathrm{X}$ and $\mathrm{Y}$ between female and male offspring (Additional file 1: Figure S2). The difference in mapped reads on chromosomes $\mathrm{X}$ and $\mathrm{Y}$ was clearly distinguishable between male and female samples with minimal background noise observed on chromosome $\mathrm{Y}$ from female samples. Upon examination of the chromosomal distribution of windows with significant differential methylation applying the same criteria employed in the exposure comparison strategy outlined in the Methods, 263 and 325 windows (total $=696$ ) were located on chromosome $\mathrm{X}$ and $\mathrm{Y}$, respectively, compared with only 108 windows on autosomes (Additional file 1: Figure S3). Despite the presence of a limited number of background reads on chromosome $\mathrm{Y}$ in female samples, no regions on this chromosome were identified to harbor hypermethylation in female samples. This analysis provides us an estimate of the maximum false discovery rate (FDR) of $15.5 \%$ $(108 / 696 * 100 \%)$ for our analysis presented below; however, the actual FDR may be much lower, if true autosomal differences in methylation exist between sexes.

\section{BPA Exposure Dependent Regions of Altered Methylation (RAMs)}

When genome-wide DNA methylation patterns were compared across BPA exposure categories (control vs. MG, control vs. UG, and UG vs. MG), a small percentage $(0.2$ to $0.3 \%)$ of windows $(106,765,166,437$, and 173,823 windows, respectively, in over 53 million windows of 100 bp with 50 bp moving-shifts) were identified as preliminary regions of altered methylation (RAMs) ( $\mathrm{p}$-value $<0.05$; Additional file 1: Figure S4A) prior to applying additional filtering steps described in Methods. Across the three BPA exposure comparisons, a majority of 
RAMs (82\%, which is 310,024 out of 378,371 in 100 bp windows) were distinct from one another (Additional file 1: Figure S4B). RAMs were identified both within and outside of CGIs, CGI shores (0-2 kb from CGI), and CGI shelves (2-4 kb from CGI) (Additional file 1: Figure S4C).

To minimize the influence of a single sample in predicting RAMs, we further analyzed data with filtered RAMs that 1) exhibited methylation change in at least two samples per exposure category and 2) displayed differential methylation either in at least one out of two flanking windows (shift window size is $50 \mathrm{bp}$ ) or two $100 \mathrm{bp}$ windows within a $500 \mathrm{bp}$ stretch. These filtering steps were used for the male versus female comparison and thus are expected to result in an FDR no greater than $15.5 \%$. We then conducted a refined downstream analysis, similar to the unfiltered analysis described above. Following filtering, within each exposure comparison we observed a greater number of hypermethylated RAMs compared to hypomethylated RAMs (Figure 2A and C). The largest number of RAMs was observed when UG exposed offspring were compared to MG exposed offspring ( $\mathrm{N}=11,647$ genome-wide, and $\mathrm{N}=428$ promoter-region only, Figure 2A and $\mathrm{C}$ ). The control versus UG exposure category resulted in the smallest number of RAMs $(\mathrm{N}=$ 2,028 genome-wide, and $\mathrm{N}=93$ promoter-region only), while the control versus MG exposure category resulted in 5772 genome-wide and 227 promoter region RAMs (Figure 2A and C). Similarly to the unfiltered analysis above, across the three BPA exposure categories, RAMs were largely distinct from one another (Figure $2 \mathrm{~B}$ and D).

Using the filtered dataset, we also examined the distribution of RAMs among CGIs, CGI shores (0-2 kb from CGI), and CGI shelves (2-4 kb from CGI), and compared these to the proportion of the M-NGS library and mouse Igenome (mm9) covered by CGIs, CGI shores, and CGI shelves. The distribution of CGIs, CGI shores, and shelves in the mouse genome ( $\mathrm{mm} 9)$ is shown in Additional file 1: Figure S5, where less than half of the genome was shown to be associated with CGIs, CGI shores, or shelves. In the M-NGS libraries enriched for CGs, over $85 \%$ of the reads were associated with CGIs, CGI shores, or shelves (43\% in CGI shores, $27 \%$ in CGIs, and $16 \%$ in CGI shelves). Less than $15 \%$ of the reads were located outside of CGIs and their surrounding area (14\%) (Figure 2E). Approximately half of the total differential regions were located within CGI shores in the Ctr vs. MG (51\%) and the UG vs. MG (50\%) comparisons, followed by CGI shelves, which accounted for over $20 \%$ of the total differential regions (Figure 2E). In the Ctr vs. UG comparison, however, a smaller proportion of the differential regions were located within CGI shores (38\%) and shelves (15\%) (Figure 2E). The relative distribution of CGIs, shores, and shelves of the RAMs in comparison with the M-NGS library identified a slight enrichment of RAMs in CGI shores (18.6\% increase in Ctr vs. MG and $16.3 \%$ increase in UG vs. MG) and CGI shelves (43.7\% increase in Ctr vs. MG and $37.5 \%$ increase in UG vs. MG), and depletion of RAMs (63\% decrease in Ctr vs. MG and UG vs. MG, and $78 \%$ decrease in Ctr vs. UG) in CGIs. In the Ctr vs. UG comparison, the relative distribution was decreased in CGI shores by $11.6 \%$, in contrast with Ctr vs. MG and UG vs. MG comparisons with an increase in relative distribution of CGI shores. These results identified the CGI shores and shelves to be the more susceptible and CGIs to be more resistant to methylation changes upon environmental exposure. Additional pie charts in Additional file 1: Figure S5 display the proportion of hyper- and hypo-methylated regions with respect to CGIs, CGI shores, and shelves.

In addition, we examined the distribution of epigenetic changes within various genomic locations including exons, 5' and 3' untranslated regions (UTRs), and within 1 and $5 \mathrm{~kb}$ of transcription start sites (TSSs) upon various BPA exposures using $\mathrm{RSeQC}$ package [32]. In Ctr vs. MG and UG vs. MG analyses, the genomic distribution of differentially methylated regions showed more than 3-fold enrichment of coding sequence (CDS) exons (3.3 fold increase in Ctr vs. MG and 3.6-fold increase in UG vs. MG) compared to background levels in the mouse genome (Figure 2F). In addition, the enrichment of 5' (1.4-fold increase in Ctr vs. MG and 1.8-fold increase in UG vs. MG) and 3'UTRs (2.1-fold increase in Ctr vs. MG and 2-fold increase in UG vs. MG) and the depletion of the upstream TSSs (1.4-fold decrease in both Ctr vs. MG and UG vs. MG for $1 \mathrm{~kb}$ and 1.3-fold decrease in Ctr vs. MG for $5 \mathrm{~kb}$ ) was observed. In the Ctr vs. UG analysis, however, the genomic distribution difference between the RAMs and the mouse genome background was not observed, except for a 2-fold increase in CDS exons. Despite the small overlap of RAMs between Ctr vs. MG and UG vs. MG comparisons, the genomic and CGI distributions of the differential regions were highly similar, and unlike the Ctr vs. UG comparison, the results deviated from the genomic background.

\section{Promoter regions associated with BPA-dependent regions of altered methylation}

Differential methylation in promoter regions may play a substantial role in gene transcriptional regulation. The identified RAMs in promoter regions $(\mathrm{N}=1,065$, $\mathrm{p}$-value $<0.05)$ that occur within $\pm 1.5 \mathrm{~kb}$ from TSSs are visualized in Figure 3. Fifty-three percent of RAMs gained methylation at promoters $(\mathrm{N}=569)$, and forty-seven percent lost methylation $(\mathrm{N}=496)$ upon BPA exposure. Promoter RAMs can be further classified into types that respond to UG exposure, respond to MG exposure, or respond to both exposures. 

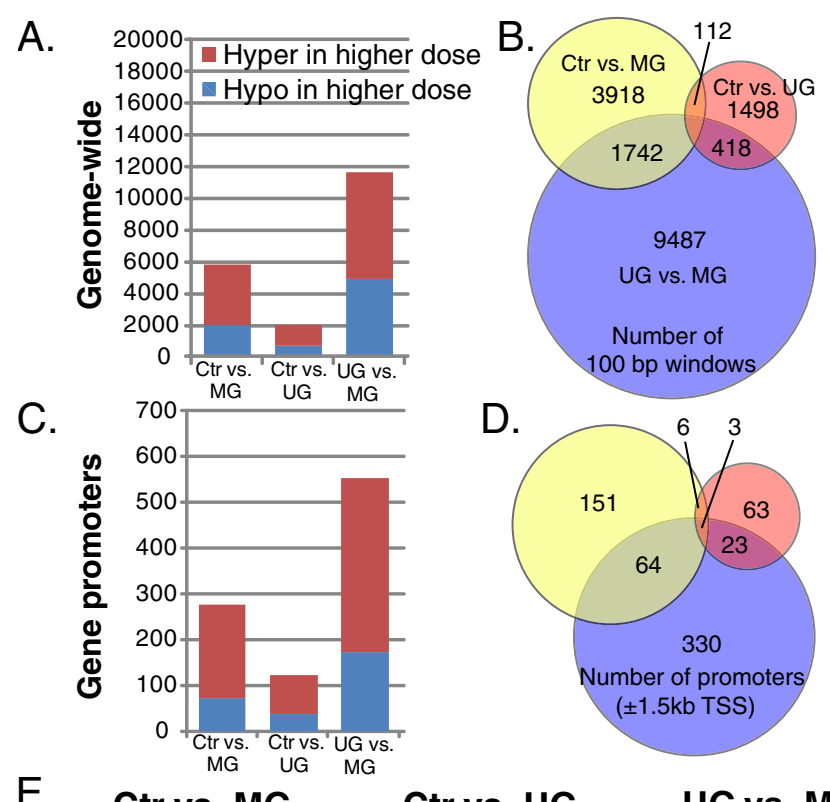

E.
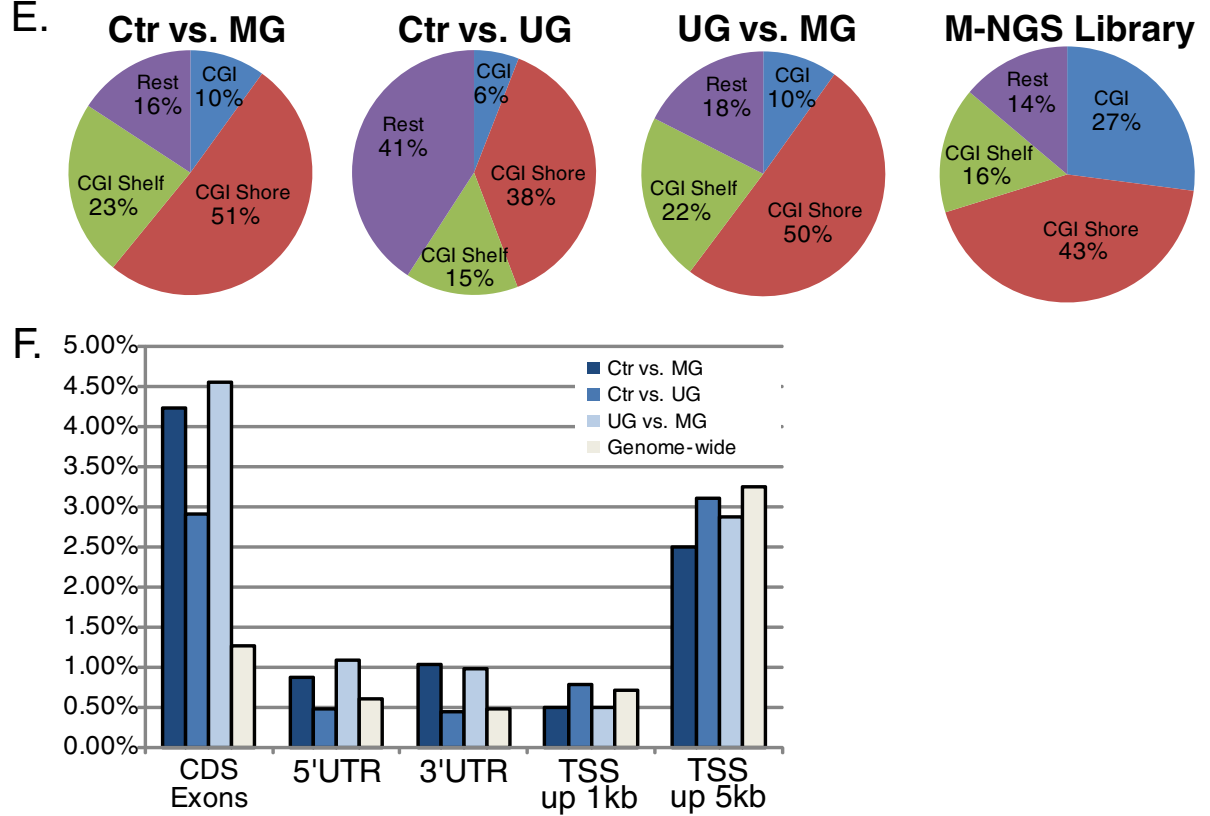

Figure 2 Characterization of genome-wide methylation in BPA-exposed offspring. The genomic distributions of regions of altered methylation (RAMs), delineated as genome-wide or within promoter regions, from the edgeR analysis with p-value $<0.05$ are displayed. (A) The bar graph identifies the number of RAMs in genome-wide scale. (B) The Venn diagram displays the overlap of RAMs among control (Ctr), $50 \mu \mathrm{g}$ (UG) and $50 \mathrm{mg}$ (MG) diet groups. (C) The bar graph and (D) Venn diagram of RAMs within promoter regions is displayed. (E) For each exposure comparison and for the full M-NGS library, pie charts display the proportion of RAMs in number of windows at CpG islands (CGIs), CpG shores, CpG shelves, and the remainder of the genome. In the Ctr vs. MG and in the UG vs. MG comparisons, approximately half of the RAMs occur in CpG shores (0-2 kb from CGIs). (F) The genomic distributions of RAMs ( $p$-value < 0.05) at 5'UTRs, TSSs, CDSs, and 3'UTRs are delineated in bar graphs. The percentage of RAMs at CDS exons in the Ctr vs. UG comparison is decreased by more than half compared to other group comparisons. At the TSSs, the percentage of RAMs is comparable among the three group comparisons.

For RAMs with a gain of methylation, only a small proportion of the TSSs were associated with the UG exposure only $(\mathrm{N}=60)$. Thus, gains of methylation upon exposure were either observed in both the UG and MG exposure groups $(\mathrm{N}=277)$ or in only the MG exposure group $(\mathrm{N}=232)$. For
RAMs with a loss of methylation, a large proportion of the TSSs were affected by the UG exposure only $(\mathrm{N}=363)$, while only a small number of TSSs showed differential methylation upon both UG and MG exposure groups $(\mathrm{N}=$ $44)$ or only in the MG exposure group $(\mathrm{N}=89)$. 


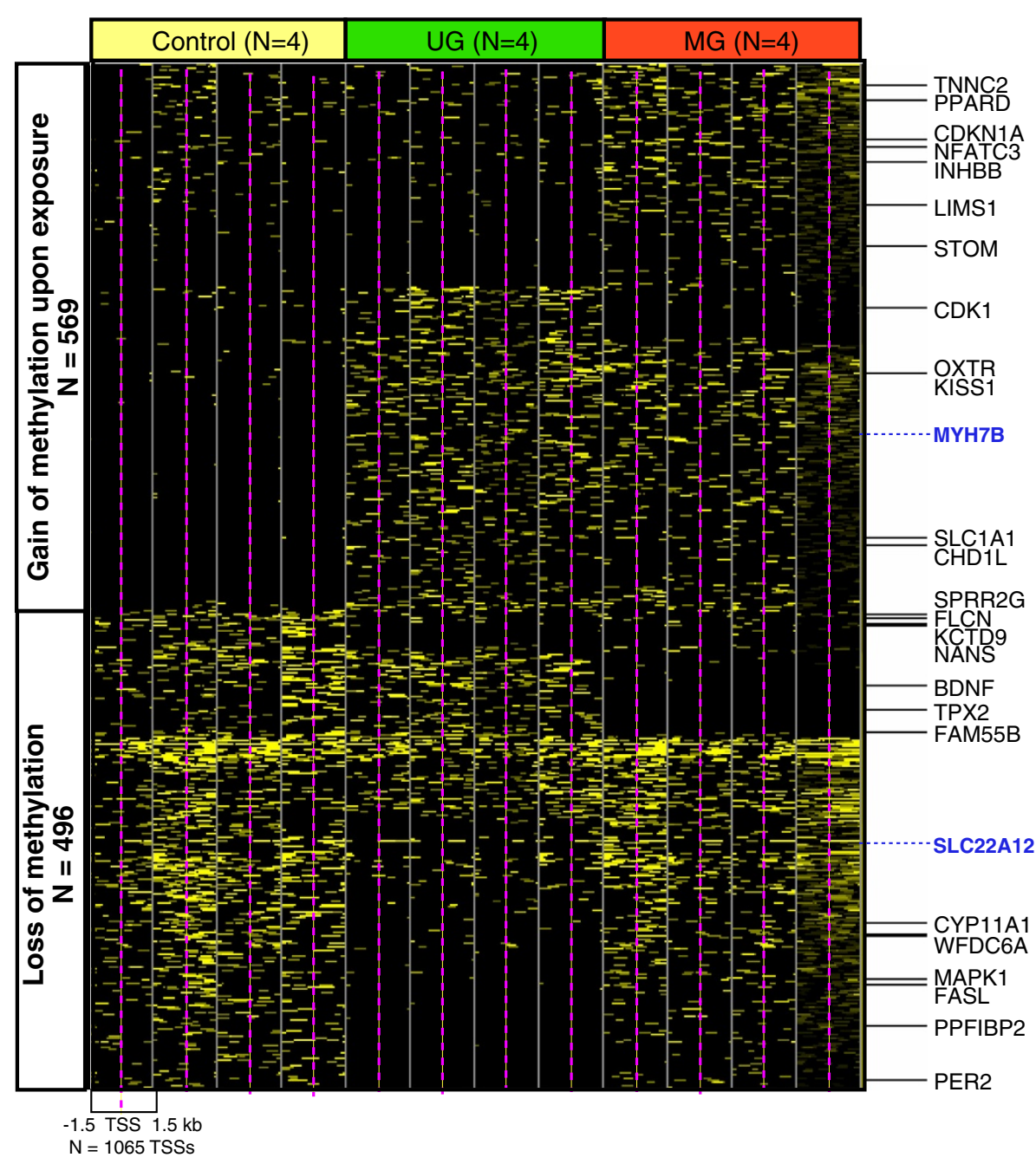

Figure 3 Regions of Altered Methylation (RAMs) in gene promoters. RAMs within $\pm 1.5 \mathrm{~kb}$ flanking transcription start sites (TSSs) are represented. Each column separated by gray lines represents a single sample (4 per group), and each row represents a unique gene promoter region, $\pm 1.5 \mathrm{~kb}$ from the TSSs (magenta dotted line). The known BPA-interacting genes queried from the Comparative Toxicogenomic Database are also indicated on the right. Two arrows pointing to the Myh7b and S/c22a12 gene promoters (in blue text) show candidate RAMs validated in a larger subset of animals.

Enriched gene ontology terms and pathways among BPA exposure dependent differentially methylated genes We examined the enrichment of Gene Ontology (GO) terms and pathways present in our candidate regions within $1.5 \mathrm{~kb}$ of a TSS $(\mathrm{N}=19,720$; Additional file 2: Table S3) using the Gene Set Enricher application from the Comprehensive Toxicogenomics Database (CTD) site, and the results were visualized using the REViGO web application (Figure 4). GO biological processes enriched for BPA-exposure RAMs in Ctr vs. MG comparison $(\mathrm{n}=198$ genes, $\mathrm{FDR}<0.05)$ included metabolism and stimulus response (Figure 4A; Additional file 2: Table S4). Only 4 significant GO molecular functions were observed, and they were involved in general binding activities (binding, protein and ion binding, catalytic activity, FDR $<0.05)$. The significant pathways altered include transmembrane transport of small molecules (REACT:15518) and metabolism (REACT:111217). In the Ctr vs. UG comparison, 76 genes were assessed, and two GO biological process terms include metabolic process and cellular process (Figure 4B). In addition, cancer related pathways were enriched (KEGG:05200) (Additional file 2: Table S4). For the UG vs MG comparison, a total of 371 genes were assessed, and we observed strong enrichment of GO terms involved in metabolic processes and stimulus as well as signaling processes (Figure 4C). The significant pathways include glutamatergic synapse (KEGG:04724) and regulation of autophagy (KEGG:04140) (Additional file 2: Table S4).

Enriched GO terms and pathway analysis was also performed on 156 known BPA-interacting genes (curated from the CTD), that are known to be expressed in the 


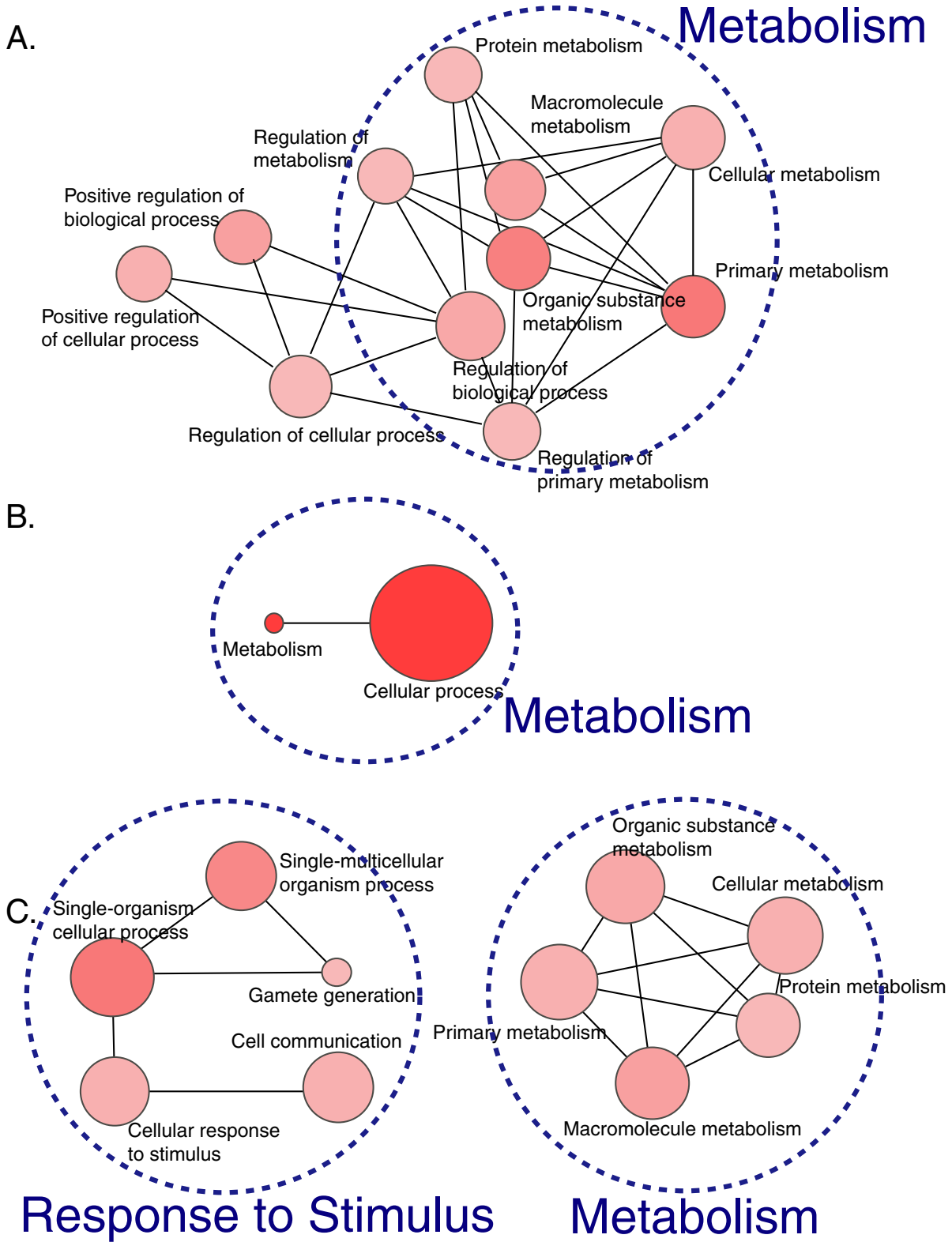

Figure 4 Enriched biological processes among genes harboring differential methylation. The genes harboring differential methylation within their promoters (FDR adjusted p-value < 0.05) were subjected to GO term enrichment analysis. (A) A total of 198 genes from Ctr vs. MG comparison, (B) 76 genes from Ctr vs. UG comparison, and (C) 371 genes from UG vs. MG comparison are used for the analysis, and enriched pathways are involved in basic biological processes, including basic cellular, metabolic, and immune and stimulus responses, as well as some binding activities. The enriched biological processes are graphed using REViGO software. The colors of the circles represent the various levels of statistical significance, where the darker shade represents more significance in p-values than the lighter one. The various sizes of the circles represent the number of genes in given $\mathrm{GO}$ terms. A complete list of $\mathrm{GO}$ terms including cellular components and molecular functions is available in Additional file 2: Table S4.

mouse liver from Mouse Genome Informatics Gene Expression Database and compared with the results from our methylation data (Additional file 2: Table S5). This analysis identified 67 pathways and 912 GO terms that are significantly enriched among BPA-interacting genes, representing genes whose altered DNA methylation may be associated with concomitant gene expression changes in the liver. Some of the top pathways include pathways in cancer (KEGG:05200, FDR < 2.77e-28), metabolism (REACT:111217, FDR <2e-21), and adipocytokine signaling pathway (KEGG:04920, FDR < 1.56e-10). By identifying enriched pathways using two lists, including one focusing on epigenetically altered genes and another on transcriptionally regulated genes, the similarities as well 
as the differences between the affected pathways via two different mechanisms can be compared.

In addition to our promoter based enrichment analysis above, whose differential sites were restricted to within $1.5 \mathrm{~kb}$ of TSSs, we also performed pathway enrichment analysis with all RAMs using ChIP-Enrich (http://chipenrich.med.umich.edu). The ChIP-Enrich application assigns peaks to genes based on a chosen method (we used nearest TSS) and tests peaks from ChIP-seq experiments for enrichment of biological pathways, GO terms, and other types of gene sets using an empirical method to adjust for the relationship between probability of a peak and the genomic length associated with a gene. Associating genomic sites or peaks to nearest TSSs has been widely applied in the biological functional analysis of ChIP-Seq data [33]. In the Ctr vs. MG and in the UG vs. MG comparisons, similar pathway enrichments were obtained as seen in our promoter region based testing, mainly metabolism and its related processes (FDR < 0.01 ), as well as GO terms related to development and morphogenesis (FDR < 0.01) (Additional file 2: Table S6). In the Ctr vs. UG enrichment results, only $7 \mathrm{GO}$ terms were significant with $\mathrm{FDR}<0.1$, while there are 109 terms enriched in Ctr vs. MG, and 119 terms enriched in UG vs. MG comparisons (FDR <0.1). Three of the top 7 enriched GO terms were lipoprotein particle receptor activity $(\mathrm{FDR}<0.04)$, low-density lipoprotein receptor activity (FDR $<0.07$ ), and apolipoprotein binding $(\mathrm{FDR}<0.09)$.

\section{Validation of regions of altered methylation using sequenom EpiTYPER}

RAMs from 5 genomic regions were quantitatively validated using the Sequenom EpiTYPER platform. We validated two RAMs located in the gene promoters of myosin, heavy chain 7B, cardiac muscle, beta $(M y h 7 b)$ (p-values $<0.006,300$ bp windows) and renal-specific transporter (Slc22a12) (p-values $<0.009,300$ bp windows) (Additional file 2: Table S7). Both of these genes were associated with metabolic process in our ChIPEnrich testing (FDR $<1.6 \mathrm{E}-4$ and $2.9 \mathrm{E}-5$ in UG vs. MG and Ctr vs. MG comparisons). Several enriched concepts involved in binding processes such as ribonucleotide, nucleotide, actin, and cytoskeletal protein bindings (FDR < 0.05) in our ChIP-Enrich analysis were associated with Myh7b, and those involved in transport activities and nitrogen metabolic process $(\mathrm{FDR}<0.05)$ were associated with Slc22a12. The methylation gain in the promoter region of $M y h 7 b$ in both the UG and MG exposures (Figure 3) was validated, showing a median methylation of 30.1\% (22.8 for Quartile 1, Q1 and 35.4 for Quartile 3, Q3) in Ctr compared to 36.8\% (32.5 for Q1 and 38.3 for Q3) in UG and 38.1\% (34.3 for Q1 and 48.6 for Q3) in MG (Figure 5A). The gene expression change in $M y h 7 b$ was monitored using real-time $\mathrm{qPCR}$, revealing no change in expression in PND22 mouse livers. The hypomethylation in the $S l c 22 a 12$ promoter region in the UG exposure group (Figure 3) was confirmed with a median methylation level across four CpG sites observed at 90\% (89.5 for Q1 and 92 for Q3) in Ctr, 84\% (82.1 for Q1 and 88.8 for Q3) in UG, and 89\% (85.5 for Q1 and 92.9 for Q3) in MG. The decrease in methylation at CpG site 2 in UG exposure group was statistically significant compared to $\mathrm{Ctr}$ ( $\mathrm{p}$-value $<0.004$ ) and MG (p-value <0.01) groups (Figure 5B). The gene expression change in $S l c 22 a 12$ was assayed via $\mathrm{qPCR}$, revealing that this gene is not expressed in PND22 mouse liver tissue.

Additionally, we measured methylation in three RAMs in intergenic regions from chromosome 1,4 , and 18 to confirm differential methylation associated with BPA exposure. Hypomethylation was validated in the region from chromosome 18 (p-value < 0.036) (Figure 5C), with a median methylation level of $82.5 \%$ (77.1 for Q1 and 86 for Q3) in the Ctr group compared to $72.5 \%$ (68 for Q1 and 77 for Q3) in UG and MG BPA group. The intergenic region from chromosome 4 was hypermethylated as indicated by the M-NGS (p-value $<0.035$ ) with a median methylation of 86\% (79 for Q1 and 98 for Q3) in the Ctr group, compared to 97.5\% (92 for Q1 and 100 for Q3) in the UG BPA group, and 95.0\% (91 for Q1 and 97.5 for Q3) in the MG group (Figure 5D). The remaining region on chromosome 1 that showed a M-NGS identified loss of methylation with BPA exposure was not differentially methylated following bisulfite sequencing validation ( $\mathrm{p}$-value $>0.35$ ), with a median methylation of $85.7 \%$ (77.7 for Q1 and 90.8 for Q3) in Ctr, 82.7\% (81.7 for Q1 and 85.3 for Q3) in UG, and 87\% (84.7 for Q1 and 91 for Q3) in MG groups.

\section{Discussion}

We have previously shown that BPA exposure at $50 \mathrm{mg}$ $\mathrm{BPA} / \mathrm{kg}$ diet during development plays a role in epigenetic programming at candidate metastable loci $A^{v y}$ and $\operatorname{Cabp}^{I A P}$ [4]. In a follow-up study using multiple doses of dietary BPA exposures, we observed dose-dependent effects on DNA methylation at $A^{v y}$ and $C a b p^{I A P}$ with the lower doses $(50 \mu \mathrm{g}$ and $50 \mathrm{ng} \mathrm{BPA} / \mathrm{kg}$ diet) leading to the opposite, hypermethylating effect [5]. We have now employed a next-generation sequencing approach and identified non-monotonic effects on the DNA methylome following human physiologically relevant perinatal BPA exposures. The identification of low dose and nonmonotonic effects of endocrine disrupting chemicals, such as BPA, is a topic of growing interest in toxicology and endocrinology [15].

Genome-wide platforms allow for identification of the constellation of genomic loci with altered epigenetic status following exposure or in relation to disease status. 


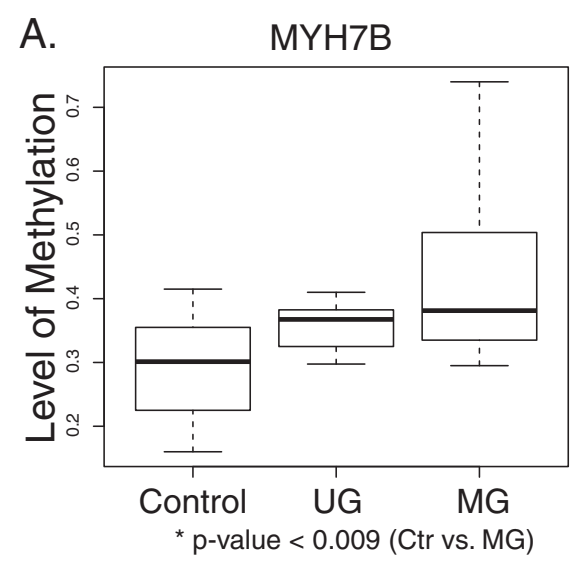

C.

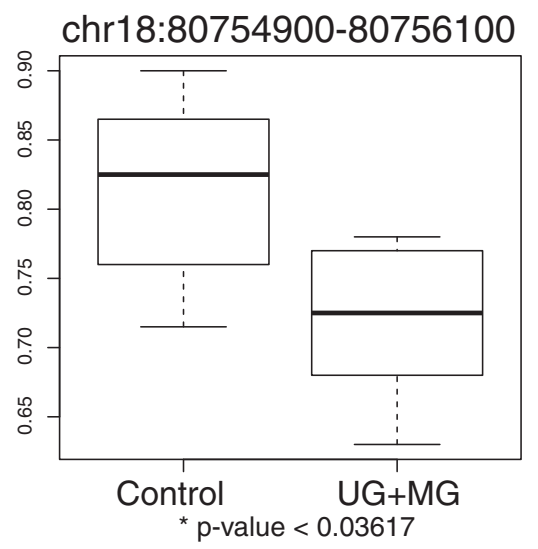

B.

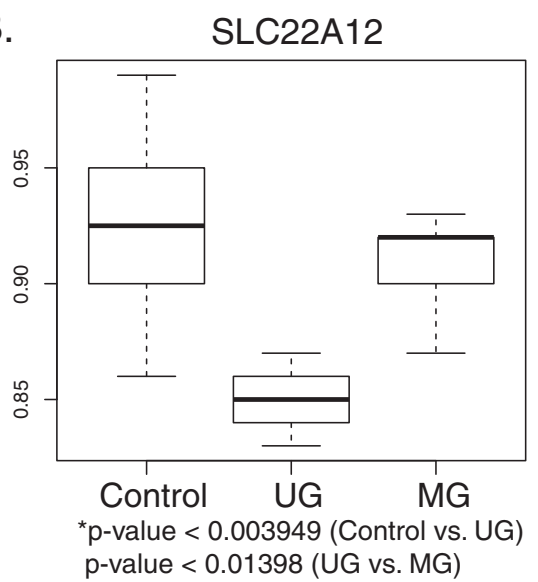

D.

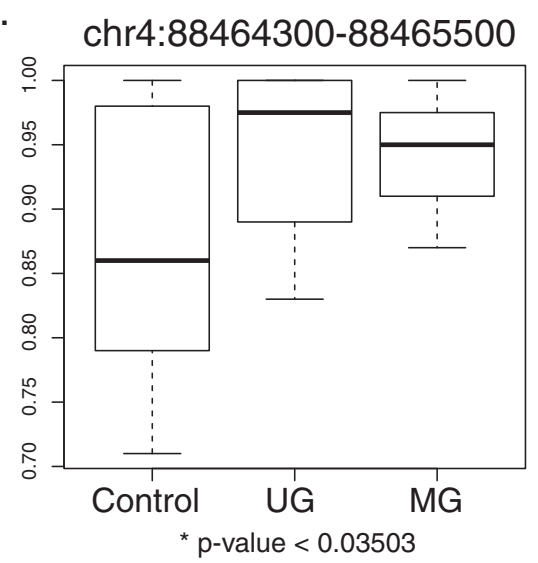

Figure 5 Candidate region validation using Sequenom EpiTYPER. RAMs from candidate regions including (A) Myh7b and (B) S/c22a12 promoters and (C, D) two intergenic regions from 29 mouse liver samples including the 12 original samples sequenced for M-NGS were quantitatively validated via Sequenom EpiTYPER and shown as box plots.

For example, Irizarry et al. used a microarray approach and demonstrated that approximately $70 \%$ of methylation changes in colon cancer samples occurred in "shores" defined as regions up to $2 \mathrm{~kb}$ away from CpG islands [34]. Newer approaches to query the methylome now involve next-generation sequencing, and the technology in this field is rapidly advancing, including whole genome and reduced representation bisulfite sequencing, which quantitatively measure methylation changes at single-based resolution, yet remain costly in addition to the need for complex bioinformatics pipelines. An alternative to bisulfite conversion approaches include affinity enrichment techniques (e.g. MeDIP-seq and MBD-seq) that involve the enrichment of methylated regions via methylation-targeted antibody or protein. These methods, however, are sensitive to antibody lot that may lead to inconsistent enrichment between experiments. In this project, we employed the MethylPlexNext Generation Sequencing (M-NGS) platform, which uses enzymatic enrichment to identify regions of altered methylation and requires only $50 \mathrm{ng}$ of starting genomic material. Because the exact composition of the enzymes used for the methylation enrichment is proprietary information (Patent Number US 2007/0031858 A1), we assessed CG enrichment prior to downstream analysis and confirmed an average of 2.3 fold CG enrichment across our 12 study samples compared to mouse reference genomes using this technology. In addition, we have previously assessed and published on the performance of the MethylPlex platform on CG enrichment in prostate cancer cell lines and tissues [35].

Our genome-wide analysis of liver DNA from mouse offspring exposed to BPA indicates that DNA methylation patterns exhibit non-monotonic effects following perinatal exposure to BPA, corroborating previous studies using multiple doses of BPA with non-monotonic outcomes [36,37]. We observed an enrichment of RAMs in CGI shores, accounting for nearly half of the identified RAMs in MG BPA group compared to either control or UG BPA groups. This suggests that CGI shores and regions outside of often-profiled $\mathrm{CpG}$ islands may be more susceptible to epigenetic changes following 
perinatal exposures. For example, RAMs identified in the higher BPA exposure group (MG) were more likely to be located within CGI shores, and CGIs were more resistant to epigenetic change. In the lower BPA exposure group (UG), however, an enrichment of the RAMs among CGI shores was not observed; instead regions with low CG density were highly enriched as RAMs. In addition, the overall distribution of the RAMs within CDS, UTRs, and TSSs was also distinct between MG and UG exposed samples.

In order to identify gene promoters with altered DNA methylation upon exposure, we scanned $\pm 1,500$ bps flanking the TSSs of 30,637 transcripts in the mouse genome (mm9). This analysis indicated distinct exposuredependent methylation patterns around TSSs (span of 3,000 bp) and identified several hundred novel BPAinduced promoter methylation events. Several of the identified promoter methylation events occurred in genes previously associated with transcriptional change following BPA exposure, including Hmgn5, Hpcal1, Hoxa10, Brca1, Pde4d [27], and Esr1 and Esr2 [29]. In addition, decreased promoter methylation and increased expression were reported in high mobility group nucleosome binding domain 5 (Hmgn5) from the prostate of male adult rat neonatally exposed to $10 \mu \mathrm{g} \mathrm{BPA} / \mathrm{kg}$ diet [30], and in Homeobox protein Hox-A10 (Hoxa10) from the reproductive tract of CD-1 mice neonatally exposed to $5 \mathrm{mg}$ $\mathrm{BPA} / \mathrm{kg}$ diet [38]. Increased promoter methylation and decreased expression in Hippocalcin-like protein 1 (Hpcal1) in new born male rats exposed to $10 \mu \mathrm{g}$ BPA $/ \mathrm{kg}$ diet [30] and breast cancer type 1 susceptibility protein (BRCA1) in human mammary epithelial cells exposed to BPA for 1 week at the early passage [39] have also been reported. Using the Comparative Toxicogenomics Database (CTD), we identified 25 genes with previously reported changes in gene expression upon BPA exposure that also harbored aberrant DNA methylation near promoters in our BPA-exposed mouse liver samples (Figure 3).

To perform technical validation as well as to identify true differential methylation target genes upon BPA exposure, the original 12 samples along with 17 additional samples were included in the validation set. Two of the validation loci were located within gene promoter regions, and thus an alteration in methylation upon BPA exposure may result in concomitant gene expression changes. One of our candidate genes that gained methylation upon BPA exposure in our M-NGS data was Myh7b. Quantitative and CpG site specific validation using the Sequenom EpiTYPER platform confirmed the increase in DNA methylation within the promoter region of $M y h 7 b$ in a monotonic dose-dependent manner (e.g. the higher the BPA exposure, the higher the methylation level). The MYH7B protein is known to interact with ESR2 [40], and one of the MYH7B estrogen- response elements (ERE) (www.genomatix.de) is located within an identified RAM. Despite the validated quantitative change in methylation in the $M y h 7 b$ promoter, no exposure dependent alteration in expression was observed in PND22 mouse liver samples. During development, genes exhibit unique time windows of expression, and it's possible a change in expression may have been missed or could occur at a future time point. Alternatively, the observed altered methylation upon BPA exposure may merely be an effect on the epigenome that will not manifest itself in a change in expression, protein level, or protein activity. Slc22a12 is a candidate RAM displaying decreased level of methylation upon BPA exposure. In humans, the presence of single nucleotide polymorphisms (SNPs) in the SLC22A12 gene was found to be associated with obesity and metabolic syndrome in Caucasians with hypertension [41]. As in the M-NGS data, a significant decrease in DNA methylation was observed in samples in the UG exposure group, but not in the MG exposure group, adding to the weight of evidence supporting non-monotonic epigenetic responses following BPA exposure.

Our pathway analysis indicated strong enrichment of genes involved in metabolism and stimulus response upon BPA exposure. This observation, in combination with previously reported data supporting a role for BPA in immune [42-45] and metabolic response [6,46-49], indicates the importance of changes in epigenetic pathways following perinatal exposures as a mechanism linking developmental exposures to disease risk in adulthood. For example, the activity of the adiponectin gene, which codes for a hormone controlling insulin sensitivity, was previously shown to be suppressed by BPA [49], implicating BPA in the development of type 2 diabetes. Stimulus response upon BPA exposure was previously identified in a prenatally BPA-exposed mouse with increased regulation of $\mathrm{T}$ helper 1 and 2 immune responses [43]. Estrogen is a known regulator of the immune response through various activities including the secretion of interferon- $\gamma$ and cytokine [50,51]. Several immune response experiments of environmental exposures including BPA have been previously conducted; in a mouse study, female offspring of mothers exposed to $>50 \mu \mathrm{g} \mathrm{BPA} / \mathrm{kg}$ had elevated lung inflammation, compared with offspring of control dams [42]. Further, prenatal exposure to $10 \mu \mathrm{g} \mathrm{BPA} / \mathrm{mL}$ in drinking water enhanced allergic sensitization and bronchial inflammation and responsiveness in a susceptible animal model of asthma [45].

To understand the full extent of BPA and associated perinatal exposures on the epigenome as a whole, it will be important to incorporate genome-wide analysis other epigenetic mechanisms such as histone modifications and non-coding RNAs, as well as full transcriptome analyses, such as RNA-seq. Indeed, we have recently 
identified DNA methylation and histone modifications to act in concert with one another at the $A^{v y}$ metastable epiallele [52]. Increasing the number of studies focusing on multiple epigenetic mechanisms will strengthen the understanding of environmentally induced alterations to the epigenome.

\section{Conclusions}

It is increasingly recognized that environmental exposure to chemical, nutritional, and behavioral factors alters gene expression and affects health and disease by not only mutating promoter and coding regions of genes, but also by modifying the epigenome. The investigation of early environmental effects can inform the fields of toxicology and environmental epidemiology by elucidating the mechanisms underlying developmental exposure and disease risk later in life. The identification of epigenomic loci dysregulated in a dose-dependent manner will ultimately strengthen human health risk assessment and shape diagnostic and therapeutic strategies for disease. The mouse is a tractable and popular model for human diseases; however animal models for toxicology studies may not be the best choice for modeling the potential impact on the human genome if the repertoire of epigenetically labile genes is markedly species dependent. Additional toxicologically relevant animal models, including rats and sheep should also be considered for this approach along with parallel approaches in human tissues. Ultimately, researchers must integrate the layers of epigenetic changes with the windows of susceptibility to understand and generate the best prescriptions for human health and disease. The comprehensive methylome map presented here will further our understanding on the methylation targets of BPA exposure. Since epigenetic profiles, unlike genetic mutations, are potentially reversible, approaches for prevention and treatment, such as nutritional supplementation and/or pharmaceutical therapies, may have significant impact on disease trajectory and, ultimately, human health.

\section{Methods}

\section{Mouse liver tissue samples}

Mice were obtained from a colony that has been maintained with sibling mating and forced heterozygosity for the viable yellow agouti $\left(A^{v y}\right)$ and non-agouti $(a)$ alleles for over 220 generations, resulting in a genetically invariant background [53]. To avoid effects associated with parity, virgin wild-type $a / a$ dams, 6 weeks of age, were randomly assigned to one of three phytoestrogen free AIN-93G diets (diet 95092 with 7\% corn oil substituted for $7 \%$ soybean oil; Harlan Teklad, Madison, WI): 1) standard diet ( $\mathrm{n}=11$ litters); 2) standard diet supplemented with $50 \mu \mathrm{g} \mathrm{BPA} / \mathrm{kg}$ diet $(\mathrm{n}=9$ litters); or 3$)$ standard diet supplemented with $50 \mathrm{mg} \mathrm{BPA} / \mathrm{kg}$ diet $(\mathrm{n}=13$ litters). All diet ingredients were supplied by
Harlan Teklad except BPA, which was supplied by NTP (National Toxicology Program, Durham NC). The MG dosage is an order of magnitude lower than the dietary administered maximum non-toxic threshold in rodents (200 mg/kg body weight/day) [54], but, it is important to note, as previously reported, that the BPA dosages capture human physiologically relevant exposure $[5,55]$.

Wild-type $a / a$ dams were provided with their respective diet two weeks prior to mating with 8-week-old $A^{v y} / a$ males and housed in polycarbonate-free cages with ad libitum access to diet and BPA-free water. The dams remained on the assigned diets throughout pregnancy and lactation, after which offspring were sacrificed at post-natal day 22 (PND22). This mating scheme produces approximately 50\% a/a genotype and 50\% $A^{v y} / a$ offspring. For this study, liver DNA from a subset of $a / a$ wild-type animals was analyzed for full methylome characteristics: 1 ) standard diet (Ctr, $\mathrm{n}=4$ offspring; 2 male and 2 female); 2) $50 \mu \mathrm{g} \mathrm{BPA} / \mathrm{kg}$ diet (UG, $\mathrm{n}=4$ offspring; 2 male and 2 female); 3) $50 \mathrm{mg} \mathrm{BPA} / \mathrm{kg} \operatorname{diet}(\mathrm{MG}, \mathrm{n}=4$ offspring; 1 male and 3 female). To validate epigenomewide DNA methylation findings, liver DNA from additional PND $22 a / a$ animals was evaluated including: 1) standard diet ( $\mathrm{n}=14$ offspring; 9 male and 5 female); 2) $50 \mu \mathrm{g} \mathrm{BPA} / \mathrm{kg}$ diet ( $\mathrm{n}=5$ offspring; 3 male and 2 female); 3) $50 \mathrm{mg} \mathrm{BPA} / \mathrm{kg}$ diet ( $\mathrm{n}=10$ offspring; 4 male and 6 female). From these mice, total genomic DNA was isolated from liver tissue using buffer ATL, proteinase $\mathrm{K}$, and RNase A (Qiagen Inc., Valencia, CA), followed by phenol-chloroform extraction and ethanol precipitation. DNA quality and concentration was assessed using a ND1000 spectrophotometer (NanoDrop Technology, Wilmington, DL).

Animals used in this study were maintained in accordance with the Guidelines for the Care and Use of Laboratory Animals (Institute of Laboratory Animal Resources, 1996) and were treated humanely and with regard for alleviation of suffering. The study protocol was approved by the University of Michigan Committee on Use and Care of Animals.

\section{M-NGS library generation}

MethylPlex library synthesis and GC-enrichment service was obtained through a commercial service at Rubicon Genomics Inc., Ann Arbor, MI (Patent Number US 2007/0031858 A1) [56]. The ability of MethylPlex combined with next-generation-sequencing (M-NGS) to identify regions of altered methylation was previously evaluated using prostate cancer cell lines and tissues, and the detail of the M-NGS methods is provided in Kim et al. [35]. Briefly, fifty nanograms of genomic DNA were digested with a proprietary cocktail of methylationsensitive restriction enzymes and then amplified by PCR with universal primers to create a MethylPlex library 
that is enriched for methylated DNA. MethylPlex DNA was then subjected to additional enzymatic treatment to deplete all non-GC-rich DNA sequences, purified and amplified in a second round of PCR. After purification, amplification adaptors were removed by a restriction enzyme digest, and the purified products were directly incorporated into the Illumina genomic DNA sequencing sample preparation kit procedure (Illumina Inc., San Diego, CA) at the end repair step, skipping the nebulization process. An adenine base was then added to the purified end repaired products using Klenow exo ( $3^{\prime}$ to $5^{\prime}$ exo minus) enzyme. The reaction product was purified, ligated to Illumina adaptors with DNA ligase and resolved on a $2 \%$ agarose gel. Gel pieces were excised at 400 base pair positions, and the DNA was extracted using Qiagen gel extraction kit (Qiagen Inc., Valencia, CA).

\section{M-NGS sequencing and alignment}

The purified MethylPlex library was analyzed by Bioanalyzer (Agilent Technologies, San Diego, CA) before subjecting it to flow cell generation, where $10 \mathrm{nM}$ of library was used to prepare flowcells with approximately 30,000 clusters per lane, with the sequencing performed by the University of Michigan DNA Sequencing Core. The raw sequencing image data obtained by Illumina GAIIx using 80 cycles of single ends were analyzed by the Illumina analysis pipeline. Around 30 million reads per sample (ranging between 27 to 37 million reads) were obtained, where approximately $70 \%$ of these were mapped uniquely to the mouse $\mathrm{mm} 9$ reference genome using BurrowsWheeler Aligner (BWA) tool (Additional file 2: Table S1).

\section{Tiered approach edge $R$ analysis}

We adopted a tiered-based profiling pipeline to identify regions of altered methylation (RAMs) by examining the locus-specific genome-wide methylation patterns associated with BPA exposure levels (Figure 1). First, we scanned the entire genome using a window size of $100 \mathrm{bp}$ with a $50 \mathrm{bp}$ moving shift, which accounts for over 53 million windows for each sample. The genomic regions containing at least 10 reads in $25 \%$ of the samples ( $\sim 4$ million windows) were then subjected to edgeR analysis, which we used to test for differences in each exposure group [57]. This step removed the regions with low reads (no methylated CGs present in our sequencing library). The edge $\mathrm{R}$ analysis using $\mathrm{R}$ software was run using the glmFit function, which uses a negative binomial generated linear model, and identified the regions with differential methylation in three different comparisons; the methylation levels from the control group $(\mathrm{n}=4)$ against the $50 \mu \mathrm{g} \mathrm{BPA} / \mathrm{kg}$ diet group (Ctr vs. UG, $\mathrm{n}=4$ ), control group against $50 \mathrm{mg} \mathrm{BPA} / \mathrm{kg}$ diet group (Ctr vs. MG, $\mathrm{n}=4$ ), and $50 \mu \mathrm{g} \mathrm{BPA} / \mathrm{kg}$ diet group against $50 \mathrm{mg} \mathrm{BPA} / \mathrm{kg}$ diet group (UG vs. MG). For downstream analysis, identified RAMs were restricted to those that are present in at least half of the samples per exposure group with a differential methylation span of at least 2 adjacent windows (span of $150 \mathrm{bp}$ ) or 2 non-adjacent windows (span of $200 \mathrm{bp}$ ) within a genomic distance of $500 \mathrm{bp}$. The above filtering step was performed to minimize the sample-specific methylation variation affecting the results. In addition, using the methylation reads mapped to chromosome $\mathrm{X}$ and $\mathrm{Y}$, the underlying methylation difference among male and female samples was distinguished and re-confirmed the sex of each mouse sample ( 5 male vs. 7 female). Using the $\mathrm{mm} 9$ Refseq annotation available from the UCSC genome browser, the gene promoters and microRNA loci within RAMs were scanned using BEDtools and in-house perl script. The complete list of RAMs and associated gene promoters and microRNA loci is available in Additional file 2: Table S3. The promoter methylation RAMs $(\mathrm{N}=1,065$, p-value $<0.05)$ that occur within $\pm 1.5 \mathrm{~kb}$ from TSSs (mm9 Refseq) containing either low reads in at least one exposure group or at least a 5-fold change in methylation reads between any two exposure groups, were visualized using a heatmap.

\section{Gene set enrichment testing}

The results from edgeR analysis after applying filters and removing sample-specific methylation variation resulted in 225 (Ctr vs. MG), 96 (Ctr vs. UG), and 421 (UG vs. MG) unique genes (p-value $<0.05$ ) harboring RAMs within $\pm 1.5 \mathrm{~kb}$ from TSSs. These represent the list of genes displaying altered methylation at each BPA exposure. The GO term and pathway enrichment analysis was performed using Gene Set Enricher from Comparative Toxicogenomics Database (CTD) using corrected p-value threshold of $0.05[58,59]$. A total of 60,9 , and 56 GO terms (in Ctr vs. MG, Ctr vs. UG, and UG vs. MG comparisons, respectively) were enriched, and the results were visualized using Reduce and Visualize Gene Ontology (REViGO) web application (revigo.irb.hr), which removed redundant GO terms and linked highly similar GO terms with the similarity cutoff value of 0.5 using the Mus musculus database [60]. Enriched GO terms and pathway analysis was also performed on the 156 known BPA-interacting genes (curated from the CTD) that are expressed in the mouse liver, obtained from the Mouse Genome Informatics Gene Expression Database using a corrected p-value of 0.01 . Genome-wide region enrichment of GO terms was performed using ChIPEnrich application using all genomic-regions (p-value < 0.05 ) that passed the filter for eliminating samplespecific methylation variation described above. Genomewide region enrichment of GO terms and pathways was performed using ChIP-Enrich (http://sartorlab.ccmb.med. umich.edu/chip-enrich) package available in $\mathrm{R}$ software 
with the nearest TSS locus definition and mouse assembly (mm9) on all genomic-regions that passed the filter for eliminating sample-specific methylation variation described above.

\section{Quantitative methylation validation}

Top candidate regions were selected based on various factors, including $\mathrm{p}$-values, the number of samples with RAMs, the number of reads, and the methylation status of adjacent regions. Among the five candidate regions selected for validation, two were located within $\pm 1.5 \mathrm{~kb}$ of TSSs. Genomic DNA from liver tissue from postnatal day (PND) $22 a / a$ mouse samples $(\mathrm{N}=29)$, including the samples that were sequenced using M-NGS in this study, were bisulfite treated using the EpiTect bisulfite kit (Qiagen Inc., Valencia, CA) to allow for the conversion of unmethylated cytosines to uracil (read as thymine during PCR amplification), whereas the methylated cytosines remain unconverted [7]. Bisulfite converted DNA was then amplified using Bio-Rad (Model \#C1000) thermal cyclers (see Additional file 2: Table S7 for primer information and PCR conditions). Amplified products were subjected to the Sequenom EpiTYPER platform (Sequenom, San Diego, CA), performed in the University of Michigan DNA Sequencing Core. For each primer set, the methylation percentage across CG sites was averaged for each sample and boxplots were used to visualize this data in Figure 5. For the primer set targeting chr18:80754900-80756100, we experienced a failed assay on 4 samples and were unable to provide boxplots with whiskers for the UG group. As the BPA exposure groups were monotonic at this locus in the M-NGS discovery stage, we pooled the UG and MG groups and used this data in Figure 5C. The differences in mean methylation levels of the samples (14 samples in Ctr, 5 in UG, and 10 in MG) in each paired group (Ctr vs. UG, Ctr vs. MG, and UG vs. MG) were tested using twotailed $\mathrm{t}$-test.

\section{Quantitative real-time $\mathrm{qPCR}$ validation}

Total RNA was isolated from 10-20 mg of frozen liver from the same set of samples assayed for quantitative methylation via the RNeasy Mini kit (Qiagen, Valencia, $\mathrm{CA}$ ) according to the manufacturer's instructions including the optional DNase digestion step. The purity and quantity of RNA was assessed using the Nanodrop 2000 spectrophotometer (Thermo Scientific, Wilmington, DE). To produce complementary DNA for each sample, $1 \mu \mathrm{g}$ of total RNA template was used with the iScript cDNA synthesis Kit (Bio-Rad, Hercules, CA) following the manufacturer's protocol. The qPCR primers for $M y h 7 b$ and Slc22a12 were designed using GenScript Real-time PCR primer design bioinformatics tools (www.genscript.com). The primer sequences for RT-qPCR were as follows: $M y h 7 b$ forward primer $5^{\prime}$-AGTTGGAGTTGTCCCAGGTC; $M y h 7 b$ reverse primer $5^{\prime}$-TG CGCCTCAGGTTAGTACAC; Slc22a12 forward primer 5'-CACGTGGGACCTGGTATGTA; Slc22a12 reverse primer 5'-CCCAAACCTATCTGAGGCAT; Gapdh forward primer 5'-TCCATGACAACTTTGGCATTG; and Gapdh reverse primer 5'-CAGTCTTCTGGGTGGCAGT GA. The thermocycler settings for cDNA synthesis included incubation at $25^{\circ} \mathrm{C}$ for $5 \mathrm{~min}, 42^{\circ} \mathrm{C}$ for $60 \mathrm{~min}$, and $90^{\circ} \mathrm{C}$ for $5 \mathrm{~min}$. Slc22a12 was not expressed in PND22 mouse liver tissue via $\mathrm{qPCR}$ analysis. This finding was confirmed via the Mouse Genomics Informatics database (www.informatics.jax.org), which reports no expression of Slc22a12 in mouse liver. The threshold cycle (CT) was obtained for target gene $M y h 7 b$ and reference CT was calculated for glyceraldehydes-3 phosphate dehydrogenase (Gapdh). Results are reported as $\Delta \mathrm{CT}$, which represents the difference between $\mathrm{CT}$ of the target gene versus the $\mathrm{CT}$ of the reference gene. The average $\Delta \mathrm{CT}$ of the Ctr exposure samples were subtracted from the average $\Delta \mathrm{CT}$ of the UG and MG exposure samples to obtain the $\Delta \Delta C T$ value, and fold change was calculated as $2^{-\triangle \Delta C T}$.

\section{CpG island (CGI) annotation}

The genomic coordinates for mouse CGIs (mm9) [61] were downloaded from UCSC Genome Browser. The genomic regions flanking up to $2 \mathrm{~kb}(0-2 \mathrm{~kb}$ from CGI) that do not overlap with nearby CGIs were defined as CGI shores. The genomic regions flanking up to $2 \mathrm{~kb}$ from CGI shores ( 2 - $4 \mathrm{~kb}$ from CGI), that do not overlap with nearby CGIs and CGI shores are defined as CGI shelves [34,62].

\section{Additional files}

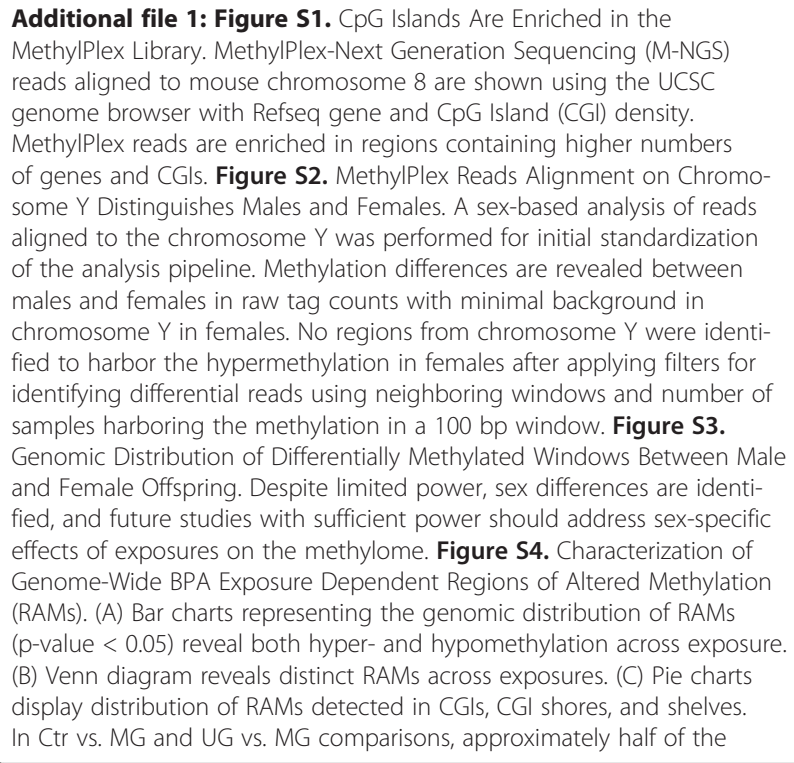


changes occur in CGI shore (0-2kb from CGI). Figure S5. Pie Charts of the Genome-wide Distribution of CGls, CGI shores, and CGI shelves in the (A) Mouse Genome and (B) Hypo and Hypermethylated Regions of Altered Methylation (RAMs). The majority of methylation changes observed in BPA-exposed mouse liver samples were located in CGI shores.

Additional file 2: Table S1. Sample table. Table S2. Gender-based differentially methylation regions. Table S3. Candidate regions. Table S4. Enriched GO terms and pathways in differentially methylated regions. Table S5. Enriched GO terms and pathways of BPA-interacting genes. Table S6. ChIP-Enrich analysis results. Table S7. EpiTYPER primers and PCR conditions.

\section{Abbreviations}

BPA: Bisphenol A; CGI: CpG islands; CTD: Comparative toxicogenomics database; CTR: Control (0 BPA exposure group); GD: Gestational day; M-NGS: MethylPlex-next generation sequencing; NHANES: National health and nutrition examination surveys; PND: Post-natal day; RAM: Region of altered methylation; TSS: Transcription start site; UG: $\mu g$ BPA exposure group; MG: mg BPA exposure group.

\section{Competing interests}

The authors declare that they have no competing interests.

\section{Authors' contributions}

$J K, M A S, L S R$, and DCD conceived of the study question and design. OSA, TRJ, MSN, and CF oversaw animal husbandry, performed DNA isolation, and assisted with MethylPlex library preparation. JK and MAS, with input from $L S R, C F$, and DCD, developed and carried out the bioinformatics pipeline to identify regions of altered methylation and test for enriched pathways. JK and DCD drafted the manuscript and created the figures with editorial guidance from MAS, LSR, and CF. All authors read and approved the final manuscript.

\section{Acknowledgements}

This work was supported by NIH grant R01 ES017524 and the University of Michigan NIEHS Center of Excellence P30 ES017885. Support for CF and MSN was provided by Institutional Training Grant T32 ES007062. The authors would like to thank the University of Michigan DNA Sequencing Core for assistance with sample processing and Rubicon Genomics (Ann Arbor, MI) for access to MethylPlex technology.

\section{Author details}

'Department of Environmental Health Sciences, University of Michigan, 1415 Washington Heights, Ann Arbor, Michigan, USA. ${ }^{2}$ Department of Computational Medicine and Bioinformatics, Medical School, University of Michigan, Ann Arbor, Michigan, USA. ${ }^{3}$ Department of Otolaryngology, Medical School, University of Michigan, Ann Arbor, Michigan, USA.

Received: 25 April 2013 Accepted: 10 January 2014

Published: 17 January 2014

\section{References}

1. Barker DJ, Eriksson JG, Forsen T, Osmond C: Fetal origins of adult disease: strength of effects and biological basis. Int J Epidemiol 2002, 31(6):1235-1239.

2. Bateson P, Barker D, Clutton-Brock T, Deb D, D'Udine B, Foley RA, Gluckman P, Godfrey K, Kirkwood T, Lahr MM, et al: Developmental plasticity and human health. Nature 2004, 430(6998):419-421.

3. Rakyan VK, Blewitt ME, Druker R, Preis JI, Whitelaw E: Metastable epialleles in mammals. Trends Genet 2002, 18(7):348-351.

4. Dolinoy DC, Huang D, Jirtle RL: Maternal nutrient supplementation counteracts bisphenol A-induced DNA hypomethylation in early development. Proc Natl Acad Sci USA 2007, 104(32):13056-13061.

5. Anderson OS, Nahar MS, Faulk C, Jones TR, Liao C, Kannan K, Weinhouse C, Rozek LS, Dolinoy DC: Epigenetic responses following maternal dietary exposure to physiologically relevant levels of bisphenol A. Environ Mol Mutagen 2012, 53(5):334-342.

6. Anderson OS, Peterson KE, Sanchez BN, Zhang Z, Mancuso PM, Dolinoy DC: Perinatal bisphenol A exposure promotes hyperactivity, lean body composition, and hormonal responses across the murine life-course. FASEB J 2013, 27(4):1784-1792.

7. Grunau C, Clark S, Rosenthal A: Bisulfite genomic sequencing: systematic investigation of critical experimental parameters. Nucl Acids Res 2001, 29(13):E65-65

8. Vandenberg LN, Chahoud I, Heindel JJ, Padmanabhan V, Paumgartten FJ, Schoenfelder G: Urinary, circulating, and tissue biomonitoring studies indicate widespread exposure to bisphenol A. Environ Health Perspect 2010, 118(8):1055-1070.

9. Calafat A, Ye X, Wong L, Reidy J, Needham L: Exposure of the U.S. population to bisphenol A and 4-tertiary-octylphenol: 2003-2004. Environ Health Perspect 2008, 116(1):39-44.

10. Zhang Z, Alomirah H, Cho HS, Li YF, Liao C, Minh TB, Mohd MA, Nakata H, Ren N, Kannan K: Urinary bisphenol A concentrations and their implications for human exposure in several Asian countries. Environ Sci Technol 2011, 45(16):7044-7050.

11. Nahar MS, Soliman AS, Colacino JA, Calafat AM, Battige K, Hablas A, Seifeldin $I A$, Dolinoy DC, Rozek LS: Urinary bisphenol A concentrations in girls from rural and urban Egypt: a pilot study. Environ Health 2012, 11:20.

12. Padmanabhan V, Siefert K, Ransom S, Johnson T, Pinkerton J, Anderson L, Tao L, Kannan K: Maternal bisphenol-A levels at delivery: a looming problem? J Perinatol 2008, 28(4):258-263.

13. Nahar MS, Liao C, Kannan K, Dolinoy DC: Fetal liver bisphenol a concentrations and biotransformation gene expression reveal variable exposure and altered capacity for metabolism in humans. J Biochem Mol Toxicol 2013, 27(2):116-123.

14. Maffini MV, Rubin BS, Sonnenschein C, Soto AM: Endocrine disruptors and reproductive health: the case of bisphenol-A. Mol Cell Endocrinol 2006, 254-255:179-186.

15. Vandenberg LN, Colborn T, Hayes TB, Heindel JJ, Jacobs DR Jr, Lee DH, Shioda T, Soto AM, Vom Saal FS, Welshons WV, et al: Hormones and endocrine-disrupting chemicals: low-dose effects and nonmonotonic dose responses. Endocr Rev 2012, 33(3):378-455.

16. Lang IA, Galloway TS, Scarlett A, Henley WE, Depledge M, Wallace RB, Melzer D: Association of urinary bisphenol A concentration with medical disorders and laboratory abnormalities in adults. JAMA 2008, 300 (11):1303-1310.

17. Meeker JD, Ehrlich S, Toth TL, Wright DL, Calafat AM, Trisini AT, Ye X, Hauser B: Semen quality and sperm DNA damage in relation to urinary bisphenol $\mathrm{A}$ among men from an infertility clinic. Reprod Toxicol 2010, 30(4):532-539.

18. Sugiura-Ogasawara M, Ozaki Y, Sonta S, Makino T, Suzumori K: Exposure to bisphenol A is associated with recurrent miscarriage. Hum Reprod 2005, 20(8):2325-2329.

19. Braun JM, Kalkbrenner AE, Calafat AM, Yolton $K$, Ye X, Dietrich KN, Lanphear BP: Impact of early-life bisphenol A exposure on behavior and executive function in children. Pediatrics 2011, 128(5):873-882.

20. Gould JC, Leonard LS, Maness SC, Wagner BL, Conner K, Zacharewski T, Safe S, McDonnell DP, Gaido KW: Bisphenol A interacts with the estrogen receptor alpha in a distinct manner from estradiol. Mol Cell Endocrinol 1998, 142(1-2):203-214.

21. Kuiper GG, Lemmen JG, Carlsson B, Corton JC, Safe SH, van der Saag PT, van der Burg B, Gustafsson JA: Interaction of estrogenic chemicals and phytoestrogens with estrogen receptor beta. Endocrinology 1998, 139(10):4252-4263.

22. Moriyama K, Tagami T, Akamizu T, Usui T, Saijo M, Kanamoto N, Hataya Y, Shimatsu A, Kuzuya H, Nakao K: Thyroid hormone action is disrupted by bisphenol A as an antagonist. J Clin Endocrinol Metab 2002, 87(11):5185-5190.

23. Thomas P, Dong J: Binding and activation of the seven-transmembrane estrogen receptor GPR30 by environmental estrogens: a potential novel mechanism of endocrine disruption. I Steroid Biochem Mol Biol 2006, 102(1-5):175-179.

24. Takayanagi S, Tokunaga T, Liu X, Okada H, Matsushima A, Shimohigashi Y: Endocrine disruptor bisphenol A strongly binds to human estrogenrelated receptor gamma (ERRgamma) with high constitutive activity. Toxicol Lett 2006, 167(2):95-105.

25. Sui Y, Ai N, Park SH, Rios-Pilier J, Perkins JT, Welsh WJ, Zhou C: Bisphenol A and its analogues activate human pregnane $\mathrm{X}$ receptor. Environ Health Perspect 2012, 120(3):399-405.

26. Kruger T, Long M, Bonefeld-Jorgensen EC: Plastic components affect the activation of the aryl hydrocarbon and the androgen receptor. Toxicology 2008, 246(2-3):112-123. 
27. Ho SM, Tang WY, Belmonte de Frausto J, Prins GS: Developmental exposure to estradiol and bisphenol $A$ increases susceptibility to prostate carcinogenesis and epigenetically regulates phosphodiesterase type 4 variant 4. Cancer Res 2006, 66(11):5624-5632.

28. Yaoi T, Itoh K, Nakamura K, Ogi H, Fujiwara Y, Fushiki S: Genome-wide analysis of epigenomic alterations in fetal mouse forebrain after exposure to low doses of bisphenol A. Biochem Biophys Res Commun 2008, 376(3):563-567.

29. Doshi T, Mehta SS, Dighe V, Balasinor N, Vanage G: Hypermethylation of estrogen receptor promoter region in adult testis of rats exposed neonatally to bisphenol A. Toxicology 2011, 289(2-3):74-82.

30. Tang WY, Morey LM, Cheung YY, Birch L, Prins GS, Ho SM: Neonatal exposure to estradiol/bisphenol A alters promoter methylation and expression of Nsbp1 and Hpcal1 genes and transcriptional programs of Dnmt3a/b and $\mathrm{Mbd} 2 / 4$ in the rat prostate gland throughout life. Endocrinology 2012, 153(1):42-55.

31. Susiarjo M, Sasson I, Mesaros C, Bartolomei MS: Bisphenol a exposure disrupts genomic imprinting in the mouse. PLoS Genet 2013, 9(4):e1003401.

32. Wang L, Wang S, Li W: RSeQC: quality control of RNA-seq experiments. Bioinformatics 2012, 28(16):2184-2185.

33. McLean CY, Bristor D, Hiller M, Clarke SL, Schaar BT, Lowe CB, Wenger AM, Bejerano G: GREAT improves functional interpretation of cis-regulatory regions. Nat Biotechnol 2010, 28(5):495-501.

34. Irizarry RA, Ladd-Acosta C, Wen B, Wu Z, Montano C, Onyango P, Cui H, Gabo K, Rongione M, Webster M, et al: The human colon cancer methylome shows similar hypo- and hypermethylation at conserved tissue-specific CpG island shores. Nat Genet 2009, 41(2):178-186.

35. Kim JH, Dhanasekaran SM, Prensner JR, Cao X, Robinson D, KalyanaSundaram S, Huang C, Shankar S, Jing X, lyer M, et al: Deep sequencing reveals distinct patterns of DNA methylation in prostate cancer. Genome Res 2011, 21(7):1028-1041.

36. Honma S, Suzuki A, Buchanan DL, Katsu Y, Watanabe H, Iguchi T: Low dose effect of in utero exposure to bisphenol $A$ and diethylstilbestrol on female mouse reproduction. Reprod Toxicol 2002, 16(2):117-122.

37. Rubin BS, Murray MK, Damassa DA, King JC, Soto AM: Perinatal exposure to low doses of bisphenol A affects body weight, patterns of estrous cyclicity, and plasma LH levels. Environ Health Perspect 2001, 109(7):675-680.

38. Bromer JG, Zhou Y, Taylor MB, Doherty L, Taylor HS: Bisphenol-A exposure in utero leads to epigenetic alterations in the developmental programming of uterine estrogen response. FASEB J 2010, 24(7):2273-2280.

39. Qin XY, Fukuda $T$, Yang L, Zaha H, Akanuma $H$, Zeng Q, Yoshinaga J, Sone $\mathrm{H}$ : Effects of bisphenol $\mathrm{A}$ exposure on the proliferation and senescence of normal human mammary epithelial cells. Cancer Biol Ther 2012, 13(5):296-306.

40. Nassa G, Tarallo R, Ambrosino C, Bamundo A, Ferraro L, Paris O, Ravo M, Guzzi PH, Cannataro M, Baumann M, et al: A large set of estrogen receptor beta-interacting proteins identified by tandem affinity purification in hormone-responsive human breast cancer cell nuclei. Proteomics 2011, 11(1):159-165.

41. Shafiu M, Johnson RJ, Turner ST, Langaee T, Gong Y, Chapman AB, Gums $J G$, Johnson JA: Urate transporter gene SLC22A12 polymorphisms associated with obesity and metabolic syndrome in caucasians with hypertension. Kidney Blood Press Res 2012, 35(6):477-482.

42. Bauer SM, Roy A, Emo J, Chapman TJ, Georas SN, Lawrence BP: The effects of maternal exposure to bisphenol $A$ on allergic lung inflammation into adulthood. Toxicol Sci 2012, 130(1):82-93.

43. Yoshino S, Yamaki K, Li X, Sai T, Yanagisawa R, Takano H, Taneda S, Hayashi $\mathrm{H}$, Mori Y: Prenatal exposure to bisphenol A up-regulates immune responses, including T helper 1 and T helper 2 responses, in mice. Immunology 2004, 112(3):489-495.

44. Yoshino S, Yamaki K, Yanagisawa R, Takano H, Hayashi H, Mori Y: Effects of bisphenol $A$ on antigen-specific antibody production, proliferative responses of lymphoid cells, and $\mathrm{TH} 1$ and $\mathrm{TH} 2$ immune responses in mice. Br J Pharmacol 2003, 138(7):1271-1276.

45. Midoro-Horiuti T, Tiwari R, Watson CS, Goldblum RM: Maternal bisphenol a exposure promotes the development of experimental asthma in mouse pups. Environ Health Perspect 2010, 118(2):273-277.

46. Batista TM, Alonso-Magdalena P, Vieira E, Amaral ME, Cederroth CR, Nef S, Quesada I, Carneiro EM, Nadal A: Short-term treatment with bisphenol-A leads to metabolic abnormalities in adult male mice. PLoS One 2012, 7(3):e33814

47. Teppala S, Madhavan S, Shankar A: Bisphenol a and metabolic syndrome: results from NHANES. Int J Endocrinol 2012, 2012:598180.

48. Vom Saal FS, Nagel SC, Coe BL, Angle BM, Taylor JA: The estrogenic endocrine disrupting chemical bisphenol A (BPA) and obesity. Mol Cell Endocrinol 2012, 354(1-2):74-84.

49. Hugo ER, Brandebourg TD, Woo JG, Loftus J, Alexander JW, Ben-Jonathan N: Bisphenol $A$ at environmentally relevant doses inhibits adiponectin release from human adipose tissue explants and adipocytes. Environ Health Perspect 2008, 116(12):1642-1647.

50. Karpuzoglu-Sahin E, Hissong BD, Ansar Ahmed S: Interferon-gamma levels are upregulated by 17-beta-estradiol and diethylstilbestrol. J Reprod Immunol 2001, 52(1-2):113-127.

51. Cutolo M, Sulli A, Seriolo B, Accardo S, Masi AT: Estrogens, the immune response and autoimmunity. Clin Exp Rheumatol 1995, 13(2):217-226.

52. Dolinoy $D$, Weinhouse $C$, Jones $T$, Rozek L, Jirtle R: Variable histone modifications at the A (vy) metastable epiallele. Epigenetics 2010, 5(7):637-644.

53. Waterland $R$, Jirtle $R$ : Transposable elements: targets for early nutritional effects on epigenetic gene regulation. Mol Cell Biol 2003, 23(15):5293-5300.

54. Takahashi O, Oishi S: Testicular toxicity of dietarily or parenterally administered bisphenol $A$ in rats and mice. Food Chem Toxicol 2003, 41(7):1035-1044.

55. Sieli PT, Jasarevic E, Warzak DA, Mao J, Ellersieck MR, Liao C, Kannan K, Collet SH, Toutain PL, Vom Saal FS, et al: Comparison of serum bisphenol A concentrations in mice exposed to bisphenol $A$ through the diet versus oral bolus exposure. Environ Health Perspect 2011, 119(9):1260-1265.

56. Makarov VL, Kamberow E, Tarrier BJ: In Isolation Of Cpg Islands By Thermal Segregation And Enzymatic Selection-amplification Method. C12P 19/34; C12Q 1/68th edition. Edited by Patent U. Ann Arbor MI (US): United States: Rubicon Genomics, Inc; 2007.

57. Robinson MD, McCarthy DJ, Smyth GK: edgeR: a Bioconductor package for differential expression analysis of digital gene expression data. Bioinformatics 2010, 26(1):139-140.

58. Davis AP, King BL, Mockus S, Murphy CG, Saraceni-Richards C, Rosenstein M, Wiegers T, Mattingly CJ: The comparative toxicogenomics database: update 2011. Nucleic Acids Res 2011, 39(Database issue):D1067-D1072.

59. Davis AP, Murphy CG, Saraceni-Richards CA, Rosenstein MC, Wiegers TC, Mattingly $C$ : Comparative toxicogenomics database: a knowledgebase and discovery tool for chemical-gene-disease networks. Nucleic Acids Res 2009, 37(Database issue):D786-D792.

60. Supek F, Bosnjak M, Skunca N, Smuc T: REVIGO summarizes and visualizes long lists of gene ontology terms. PLoS One 2011, 6(7):e21800.

61. Gardiner-Garden M, Frommer M: CpG islands in vertebrate genomes. J Mol Biol 1987, 196(2):261-282

62. Doi A, Park IH, Wen B, Murakami P, Aryee MJ, Irizarry R, Herb B, Ladd-Acosta C, Rho J, Loewer S, et al: Differential methylation of tissue- and cancer-specific CpG island shores distinguishes human induced pluripotent stem cells, embryonic stem cells and fibroblasts. Nat Genet 2009, 41(12):1350-1353.

doi:10.1186/1471-2164-15-30

Cite this article as: Kim et al.: Perinatal bisphenol $A$ exposure promotes dose-dependent alterations of the mouse methylome. BMC Genomics 2014 15:30.

\section{Submit your next manuscript to BioMed Central and take full advantage of:}

- Convenient online submission

- Thorough peer review

- No space constraints or color figure charges

- Immediate publication on acceptance

- Inclusion in PubMed, CAS, Scopus and Google Scholar

- Research which is freely available for redistribution 